\title{
Cryo-EM structure of the 2019-nCoV spike in the prefusion conformation
}

\author{
Daniel Wrapp ${ }^{1 *}$, Nianshuang Wang1*, Kizzmekia S. Corbett ${ }^{2}$, Jory A. Goldsmith", Ching-Lin Hsieh", \\ Olubukola Abiona ${ }^{2}$, Barney S. Graham², Jason S. McLellan'† \\ 1Department of Molecular Biosciences, The University of Texas at Austin, Austin, TX 78712, USA. ${ }^{2}$ Vaccine Research Center, National Institute of Allergy and Infectious \\ Diseases, National Institutes of Health, Bethesda, MD 20892, USA. \\ *These authors contributed equally to this work. \\ †Corresponding author. Email: jmclellan@austin.utexas.edu
}

The outbreak of a novel betacoronavirus (2019-nCoV) represents a pandemic threat that has been declared a public health emergency of international concern. The CoV spike (S) glycoprotein is a key target for vaccines, therapeutic antibodies, and diagnostics. To facilitate medical countermeasure (MCM) development, we determined a $3.5 \AA$-resolution cryo-EM structure of the 2019-nCoV S trimer in the prefusion conformation. The predominant state of the trimer has one of the three receptor-binding domains (RBDs) rotated up in a receptor-accessible conformation. We also show biophysical and structural evidence that the 2019-nCoV S binds ACE2 with higher affinity than SARS-CoV S. Additionally, we tested several published SARS-CoV RBD-specific monoclonal antibodies and found that they do not have appreciable binding to 2019-nCoV S, suggesting antibody cross-reactivity may be limited between the two RBDs. The structure of 2019-nCoV S should enable rapid development and evaluation of MCMs to address the ongoing public health crisis.

The novel coronavirus 2019-nCoV has recently emerged as a human pathogen in the city of Wuhan in China's Hubei province, causing fever, severe respiratory illness and pneumonia-a disease recently named COVID-19 $(1,2)$. According to the World Health Organization (WHO) on February $16^{\text {th }}$, 2020, there had been over 51,000 confirmed cases globally, leading to at least 1,600 deaths. The emerging pathogen was rapidly characterized as a novel member of the betacoronavirus genus, closely related to several bat coronaviruses as well as severe acute respiratory syndrome coronavirus (SARS$\mathrm{CoV})(3,4)$. Compared to SARS-CoV, 2019-nCoV appears to be more readily transmitted from human-to-human, spreading to multiple continents and leading to the WHO declaration of a Public Health Emergency of International Concern (PHEIC) on January $30^{\text {th }}, 2020(1,5,6)$.

2019-nCoV makes use of a densely glycosylated spike (S) protein to gain entry into host cells. The $\mathrm{S}$ protein is a trimeric class I fusion protein that exists in a metastable prefusion conformation that undergoes a dramatic structural rearrangement to fuse the viral membrane with the host-cell membrane $(7,8)$. This process is triggered when the $\mathrm{S} 1$ subunit binds to a host-cell receptor. Receptor binding destabilizes the prefusion trimer, resulting in shedding of the S1 subunit and transition of the S2 subunit to a stable postfusion conformation (9). In order to engage a host-cell receptor, the receptor-binding domain (RBD) of S1 undergoes hinge-like conformational movements that transiently hide or expose the determinants of receptor binding. These two states are referred to as the "down" conformation and the "up" conformation, where down corresponds to the receptor-inaccessible state and up corresponds to the receptor-accessible state, which is thought to be less stable (10-13). Due to the indispensable function of the S protein, it represents a target for antibody-mediated neutralization, and characterization of the prefusion S structure would provide atomic-level information to guide vaccine design and development.

Based on the first reported genome sequence of 2019$\mathrm{nCoV}(4)$, we expressed ectodomain residues 1-1208 of 2019$\mathrm{nCoV} \mathrm{S}$, adding two stabilizing proline mutations in the Cterminal S2 fusion machinery based on a previous stabilization strategy that proved effective for other betacoronavirus S proteins $(11,14)$. Figure 1A shows the domain organization of the expression construct and figure S1 shows the purification process. We obtained roughly $0.5 \mathrm{mg} / \mathrm{L}$ of the recombinant prefusion-stabilized S ectodomain from FreeStyle 293 cells, and the protein was purified to homogeneity by affinity chromatography and size-exclusion chromatography (fig. S1). Cryo-EM grids were prepared using this purified, fully glycosylated S protein and preliminary screening revealed a high particle density with little aggregation near the edges of the holes.

After collecting and processing 3,207 micrograph movies, we obtained a 3.5 $\AA$-resolution 3D reconstruction of an asymmetrical trimer in which a single RBD was observed in the up 
conformation. (Fig. 1B, fig. S2, and table S1). Due to the small size of the RBD $(\sim 21 \mathrm{kDa})$, the asymmetry of this conformation was not readily apparent until $a b$ initio $3 \mathrm{D}$ reconstruction and 3D classification were performed (Fig. 1B and fig. S3). By using the 3D variability feature in cryoSPARC v2 (15), we observed breathing of the S1 subunits as the RBD underwent a hinge-like movement, which likely contributed to the relatively poor local resolution of S1 compared to the more stable S2 subunit (movies S1 and S2). This seemingly stochastic RBD movement has been captured during structural characterization of the closely related betacoronaviruses SARS-CoV and MERS-CoV, as well as the more distantly related alphacoronavirus porcine epidemic diarrhea virus (PEDV) $(10,11,13,16)$. The observation of this phenomenon in 2019-nCoV S suggests that it shares the same mechanism of triggering that is thought to be conserved among the Coronaviridae, wherein receptor-binding to exposed RBDs leads to an unstable 3 RBD-up conformation that results in shedding of $\mathrm{S} 1$ and refolding of $\mathrm{S} 2(11,12)$.

Because the $\mathrm{S} 2$ subunit appeared to be a symmetric trimer, we performed a 3D refinement imposing C3 symmetry, resulting in a $3.2 \AA$-resolution map, with excellent density for the S2 subunit. Using both maps, we built most of the 2019$\mathrm{nCoV} \mathrm{S}$ ectodomain, including glycans at 44 of the $66 \mathrm{~N}$ linked glycosylation sites per trimer (fig. S4). Our final model spans S residues 27-1146, with several flexible loops omitted. Like all previously reported coronavirus S ectodomain structures, the density for 2019-nCoV S begins to fade after the connector domain (CD), reflecting the flexibility of the heptad repeat 2 (HR2) domain in the prefusion conformation (fig. S4A) (13, 16-18).

The overall structure of 2019-nCoV S resembles that of SARS-CoV S, with a root mean square deviation (RMSD) of $3.8 \AA$ over $959 \mathrm{C} \alpha$ atoms (Fig. 2A). One of the larger differences between these two structures (although still relatively minor) is the position of the RBDs in their respective down conformations. Whereas the SARS-CoV RBD in the down conformation packs tightly against the $\mathrm{N}$-terminal domain (NTD) of the neighboring protomer, the 2019-nCoV RBD in the down conformation is angled closer to the central cavity of the trimer (Fig. 2B). Despite this observed conformational difference, when the individual structural domains of 2019$\mathrm{nCoV} \mathrm{S}$ are aligned to their counterparts from SARS-CoV S, they reflect the high degree of structural homology between the two proteins, with the NTDs, RBDs, subdomains 1 and 2 (SD1 and SD2) and S2 subunits yielding individual RMSD values of $2.6 \AA, 3.0 \AA, 2.7 \AA$ and $2.0 \AA$, respectively (Fig. 2C).

2019-nCoV S shares $98 \%$ sequence identity with the S protein from the bat coronavirus RaTG13, with the most notable variation arising from an insertion in the $\mathrm{S} 1 / \mathrm{S} 2$ protease cleavage site that results in an "RRAR" furin recognition site in $2019-\mathrm{nCoV}$ (19), rather than the single arginine in SARS-
$\mathrm{CoV}$ (fig. S5) (20-23). Notably, in influenza viruses, amino acid insertions that create a polybasic furin site in a related position in influenza hemagglutinin proteins are often found in highly virulent avian and human influenza viruses (24). In the structure reported here, the $\mathrm{S} 1 / \mathrm{S} 2$ junction is in a disordered solvent-exposed loop. In addition to this insertion of residues in the S1/S2 junction, 29 variant residues exist between 2019-nCoV S and RaTG13 S, with 17 of these positions mapping to the RBD (figs. S5 and S6). We also analyzed the 61 available 2019-nCoV S sequences in the Global Initiative on Sharing All Influenza Data (GISAID, gisaid.org) database and found that there were only 9 amino acid substitutions among all deposited sequences. Most of these substitutions are relatively conservative and are not expected to have a dramatic effect on the structure or function of the 2019-nCoV S protein (fig. S6).

Recent reports demonstrating that 2019-nCoV S and SARS-CoV S share the same functional host-cell receptorangiotensin-converting enzyme 2 (ACE2) (22, 25-27)prompted us to quantify the kinetics of this interaction by surface plasmon resonance (SPR). ACE2 bound to 2019-nCoV $\mathrm{S}$ ectodomain with $\sim 15 \mathrm{nM}$ affinity, which is approximately 10- to 20-fold higher affinity than ACE2 binding to SARS-CoV S (Fig. 3A and fig. S7) (14). We also formed a complex of ACE2 bound to the 2019-nCoV S ectodomain and observed it by negative-stain EM, where it strongly resembled the complex formed between SARS-CoV S and ACE2, which has been observed at high-resolution by cryo-EM (Fig. 3B) $(14,28)$. The high affinity of 2019-nCoV S for human ACE2 may contribute to the apparent ease with which $2019-\mathrm{nCoV}$ can spread from human-to-human (I), however, additional studies are needed to investigate this possibility.

The overall structural homology and shared receptor usage between SARS-CoV S and 2019-nCoV S prompted us to test published SARS-CoV RBD-directed monoclonal antibodies (mAbs) for cross-reactivity to the 2019-nCoV RBD (Fig. 4A). A 2019-nCoV RBD-SD1 fragment (S residues 319-591) was recombinantly expressed, and appropriate folding of this construct was validated by measuring ACE2 binding using biolayer interferometry (BLI) (Fig. 4B). Cross-reactivity of the SARS-CoV RBD-directed mAbs S230, m396 and 80R was then evaluated by BLI $(12,29-31)$. Despite the relatively high degree of structural homology between the 2019-nCoV RBD and the SARS-CoV RBD, no binding to the 2019-nCoV RBD could be detected for any of the three mAbs at the concentration tested $(1 \mu \mathrm{M})$ (Fig. $4 \mathrm{C})$, in contrast to the strong binding we observed to the SARS-CoV RBD (fig. S8). Although the epitopes of these three antibodies represent a relatively small percentage of the surface area of the 2019-nCoV RBD, the lack of observed binding suggests that SARS-directed mAbs will not necessarily be cross-reactive and that future antibody isolation and therapeutic design efforts will benefit from using 
2019-nCoV S proteins as probes.

The rapid global spread of 2019-nCoV, prompting the PHEIC declaration by WHO signals the urgent need for coronavirus vaccines and therapeutics. Knowing the atomic-level structure of the 2019-nCoV spike will allow for additional protein engineering efforts that could improve antigenicity and protein expression for vaccine development. The structural data also facilitates the evaluation of $2019-\mathrm{nCoV}$ spike mutations that will occur as the virus undergoes genetic drift and help define whether those residues have surface exposure and map to sites of known antibody epitopes for other coronavirus spike proteins. In addition, the structure provides assurance that the protein produced by this construct is homogeneous and in the prefusion conformation, which should maintain the most neutralization-sensitive epitopes when used as candidate vaccine antigens or B cell probes for isolating neutralizing human monoclonal antibodies. Furthermore, the atomic-level detail will enable the design and screening of small molecules with fusion-inhibiting potential. This information will support precision vaccine design and discovery of anti-viral therapeutics, accelerating medical countermeasure development.

\section{REFERENCES AND NOTES}

1. J. F. Chan, S. Yuan, K.-H. Kok, K. K.-W. To, H. Chu, J. Yang, F. Xing, J. Liu, C. C.-Y. Yip, R. W.-S. Poon, H.-W. Tsoi, S. K.-F. Lo, K.-H. Chan, V. K.-M. Poon, W.-M. Chan, J. D. Ip, J.-P. Cai, V. C.-C. Cheng, H. Chen, C. K.-M. Hui, K.-Y. Yuen, A familial cluster of pneumonia associated with the 2019 novel coronavirus indicating person-toperson transmission: A study of a family cluster. Lancet 395, 514-523 (2020). doi:10.1016/S0140-6736(20)30154-9 Medline

2. C. Huang, Y. Wang, X. Li, L. Ren, J. Zhao, Y. Hu, L. Zhang, G. Fan, J. Xu, X. Gu, Z. Cheng, T. Yu, J. Xia, Y. Wei, W. Wu, X. Xie, W. Yin, H. Li, M. Liu, Y. Xiao, H. Gao, L. Guo, J. Xie, G. Wang, R. Jiang, Z. Gao, Q. Jin, J. Wang, B. Cao, Clinical features of patients infected with 2019 novel coronavirus in Wuhan, China. Lancet 395, 497506 (2020). doi:10.1016/S0140-6736(20)30183-5 Medline

3. R. Lu, X. Zhao, J. Li, P. Niu, B. Yang, H. Wu, W. Wang, H. Song, B. Huang, N. Zhu, Y. Bi, X. Ma, F. Zhan, L. Wang, T. Hu, H. Zhou, Z. Hu, W. Zhou, L. Zhao, J. Chen, Y. Meng, J. Wang, Y. Lin, J. Yuan, Z. Xie, J. Ma, W. J. Liu, D. Wang, W. Xu, E. C. Holmes, G. F. Gao, G. Wu, W. Chen, W. Shi, W. Tan, Genomic characterisation and epidemiology of 2019 novel coronavirus: Implications for virus origins and receptor binding. Lancet S0140-6736(20)30251-8 (2020). doن:10.1016/SO1406736(20)30251-8 Medline

4. F. Wu, S. Zhao, B. Yu, Y.-M. Chen, W. Wang, Z.-G. Song, Y. Hu, Z.-W. Tao, J.-H. Tian, Y.-Y. Pei, M.-L. Yuan, Y.-L. Zhang, F.-H. Dai, Y. Liu, Q.-M. Wang, J.-J. Zheng, L. Xu, E. C. Holmes, Y.-Z. Zhang, A new coronavirus associated with human respiratory disease in China. Nature (2020). doi:10.1038/s41586-020-2008-3 Medline

5. N. Chen, M. Zhou, X. Dong, J. Qu, F. Gong, Y. Han, Y. Qiu, J. Wang, Y. Liu, Y. Wei, J. Xia, T. Yu, X. Zhang, L. Zhang, Epidemiological and clinical characteristics of 99 cases of 2019 novel coronavirus pneumonia in Wuhan, China: A descriptive study. Lancet 395, 507-513 (2020). doi:10.1016/S0140-6736(20)30211-7 Medline

6. Q. Li, X. Guan, P. Wu, X. Wang, L. Zhou, Y. Tong, R. Ren, K. S. M. Leung, E. H. Y. Lau, J. Y. Wong, X. Xing, N. Xiang, Y. Wu, C. Li, Q. Chen, D. Li, T. Liu, J. Zhao, M. Li, W. Tu, C. Chen, L. Jin, R. Yang, Q. Wang, S. Zhou, R. Wang, H. Liu, Y. Luo, Y. Liu, G. Shao, H. Li, Z. Tao, Y. Yang, Z. Deng, B. Liu, Z. Ma, Y. Zhang, G. Shi, T. T. Y. Lam, J. T. K. Wu, G. F. Gao, B. J. Cowling, B. Yang, G. M. Leung, Z. Feng, Early Transmission Dynamics in Wuhan, China, of Novel Coronavirus-Infected Pneumonia. N. Engl. J. Med. NEJMoa2001316 (2020). doi:10.1056/NEJMoa2001316 Medline

7. F. Li, Structure, Function, and Evolution of Coronavirus Spike Proteins. Annu. Rev. Virol. 3, 237-261 (2016). doi:10.1146/annurev-virology-110615-042301 Medline

8. B. J. Bosch, R. van der Zee, C. A. de Haan, P. J. Rottier, The coronavirus spike protein is a class I virus fusion protein: Structural and functional characterization of the fusion core complex. J. Virol. 77, 8801-8811 (2003). doi:10.1128/JVL.77.16.88018811.2003 Medline

9. A. C. Walls, M. A. Tortorici, J. Snijder, X. Xiong, B.-J. Bosch, F. A. Rey, D. Veesler, Tectonic conformational changes of a coronavirus spike glycoprotein promote membrane fusion. Proc. Natl. Acad. Sci. U.S.A. 114, 11157-11162 (2017), doi:10.1073/pnas.1708727114 Medline

10. M. Gui, W. Song, H. Zhou, J. Xu, S. Chen, Y. Xiang, X. Wang, Cryo-electron microscopy structures of the SARS-CoV spike glycoprotein reveal a prerequisite conformational state for receptor binding. Cell Res. 27, 119-129 (2017). doi:10.1038/cr.2016.152 Medline

11. J. Pallesen, N. Wang, K. S. Corbett, D. Wrapp, R. N. Kirchdoerfer, H. L. Turner, C. A. Cottrell, M. M. Becker, L. Wang, W. Shi, W.-P. Kong, E. L. Andres, A. N. Kettenbach, M. R. Denison, J. D. Chappell, B. S. Graham, A. B. Ward, J. S. McLellan, Immunogenicity and structures of a rationally designed prefusion MERS-CoV spike antigen. Proc. Natl. Acad. Sci. U.S.A. 114, E7348-E7357 (2017). doi:10.1073/pnas.1707304114 Medline

12. A. C. Walls, X. Xiong, Y.-J. Park, M. A. Tortorici, J. Snijder, J. Quispe, E. Cameroni, R. Gopal, M. Dai, A. Lanzavecchia, M. Zambon, F. A. Rey, D. Corti, D. Veesler, Unexpected receptor functional mimicry elucidates activation of coronavirus fusion. Cell 176, 1026-1039.e15 (2019). doi:10.1016/j.cell.2018.12.028 Medline

13. Y. Yuan, D. Cao, Y. Zhang, J. Ma, J. Qi, Q. Wang, G. Lu, Y. Wu, J. Yan, Y. Shi, X. Zhang, G. F. Gao, Cryo-EM structures of MERS-CoV and SARS-CoV spike glycoproteins reveal the dynamic receptor binding domains. Nat. Commun. 8, 15092 (2017). doi:10.1038/ncomms 15092 Medline

14. R. N. Kirchdoerfer, N. Wang, J. Pallesen, D. Wrapp, H. L. Turner, C. A. Cottrell, K. S. Corbett, B. S. Graham, J. S. McLellan, A. B. Ward, Stabilized coronavirus spikes are resistant to conformational changes induced by receptor recognition or proteolysis. Sci. Rep. 8, 15701 (2018). doi:10.1038/s41598-018-34171-7 Medline

15. A. Punjani, J. L. Rubinstein, D. J. Fleet, M. A. Brubaker, cryoSPARC: Algorithms for rapid unsupervised cryo-EM structure determination. Nat. Methods 14, 290-296 (2017). doi:10.1038/nmeth.4169 Medline

16. D. Wrapp, J. S. McLellan, The 3.1-angstrom cryo-electron microscopy structure of the porcine epidemic diarrhea virus spike protein in the prefusion conformation. J. Virol. 93, e00923-19 (2019). doi:10.1128/JVI.00923-19 Medline

17. A. C. Walls, M. A. Tortorici, B. Frenz, J. Snijder, W. Li, F. A. Rey, F. DiMaio, B.-J. Bosch, D. Veesler, Glycan shield and epitope masking of a coronavirus spike protein observed by cryo-electron microscopy. Nat. Struct. Mol. Biol. 23, 899905 (2016). doi:10.1038/nsmb.3293 Medline

18. R. N. Kirchdoerfer, C. A. Cottrell, N. Wang, J. Pallesen, H. M. Yassine, H. L. Turner, K. S. Corbett, B. S. Graham, J. S. McLellan, A. B. Ward, Pre-fusion structure of a human coronavirus spike protein. Nature 531, 118-121 (2016). doi:10.1038/nature17200 Medline

19. B. Coutard, C. Valle, X. de Lamballerie, B. Canard, N. G. Seidah, E. Decroly, The spike glycoprotein of the new coronavirus 2019-nCoV contains a furin-like cleavage site absent in CoV of the same clade. Antiviral Res. 176, 104742 (2020). doi:10.1016/jantiviral.2020.104742 Medline

20. B. J. Bosch, W. Bartelink, P. J. Rottier, Cathepsin L functionally cleaves the severe acute respiratory syndrome coronavirus class I fusion protein upstream of rather than adjacent to the fusion peptide. J. Virol. 82, 8887-8890 (2008). doi:10.1128/JVI.00415-08 Medline

21. I. Glowacka, S. Bertram, M. A. Müller, P. Allen, E. Soilleux, S. Pfefferle, I. Steffen, T. S. Tsegaye, Y. He, K. Gnirss, D. Niemeyer, H. Schneider, C. Drosten, S. Pöhlmann, Evidence that TMPRSS2 activates the severe acute respiratory syndrome coronavirus spike protein for membrane fusion and reduces viral control by the humoral immune response. J. Virol. 85, 4122-4134 (2011). doi:10.1128/JVl.02232-10 Medline

22. W. Li, M. J. Moore, N. Vasilieva, J. Sui, S. K. Wong, M. A. Berne, M. Somasundaran, J. L. Sullivan, K. Luzuriaga, T. C. Greenough, H. Choe, M. Farzan, Angiotensinconverting enzyme 2 is a functional receptor for the SARS coronavirus. Nature 426, 450-454 (2003). doi:10.1038/nature02145 Medline

23. S. Belouzard, V. C. Chu, G. R. Whittaker, Activation of the SARS coronavirus spike protein via sequential proteolytic cleavage at two distinct sites. Proc. Natl. Acad. Sci. U.S.A. 106, 5871-5876 (2009). doi:10.1073/pnas.0809524106 Medline

24. J. Chen, K. H. Lee, D. A. Steinhauer, D. J. Stevens, J. J. Skehel, D. C. Wiley, Structure 
of the hemagglutinin precursor cleavage site, a determinant of influenza pathogenicity and the origin of the labile conformation. Cell 95, 409-417 (1998). doi:10.1016/S0092-8674(00)81771-7 Medline

25. M. Hoffmann, H. Kleine-Weber, N. Krüger, M. Müller, C. Drosten, S. Pöhlmann, The novel coronavirus 2019 (2019-nCoV) uses the SARS-coronavirus receptor ACE2 and the cellular protease TMPRSS2 for entry into target cells. bioRxiv 929042 [Preprint]. 31 January 2020. https://doi.org/10.1101/2020.01.31.929042.

26. Y. Wan, J. Shang, R. Graham, R. S. Baric, F. Li, Receptor recognition by novel coronavirus from Wuhan: An analysis based on decade-long structural studies of SARS. J. Virol. JVI.00127-20 (2020). doi:10.1128/JVl.00127-20 Medline

27. P. Zhou, X.-L. Yang, X.-G. Wang, B. Hu, L. Zhang, W. Zhang, H.-R. Si, Y. Zhu, B. Li, C.-L. Huang, H.-D. Chen, J. Chen, Y. Luo, H. Guo, R.-D. Jiang, M.-Q. Liu, Y. Chen, X.-R. Shen, X. Wang, X.-S. Zheng, K. Zhao, Q.-J. Chen, F. Deng, L.-L. Liu, B. Yan, F.X. Zhan, Y.-Y. Wang, G.-F. Xiao, Z.-L. Shi, A pneumonia outbreak associated with a new coronavirus of probable bat origin. Nature (2020). doi:10.1038/s41586-0202012-7 Medline

28. W. Song, M. Gui, X. Wang, Y. Xiang, Cryo-EM structure of the SARS coronavirus spike glycoprotein in complex with its host cell receptor ACE2. PLOS Pathog. 14, e1007236 (2018). doi:10.1371/journal.ppat.1007236 Medline

29. W. C. Hwang, Y. Lin, E. Santelli, J. Sui, L. Jaroszewski, B. Stec, M. Farzan, W. A. Marasco, R. C. Liddington, Structural basis of neutralization by a human antisevere acute respiratory syndrome spike protein antibody, 80R. J. Biol. Chem. 281, 34610-34616 (2006). doi:10.1074/ibc.M603275200 Medline

30. P. Prabakaran, J. Gan, Y. Feng, Z. Zhu, V. Choudhry, X. Xiao, X. Ji, D. S. Dimitrov, Structure of severe acute respiratory syndrome coronavirus receptor-binding domain complexed with neutralizing antibody. J. Biol. Chem. 281, 15829-15836 (2006). doi:10.1074/ibc.M600697200 Medline

31. X. Tian, C. Li, A. Huang, S. Xia, S. Lu, Z. Shi, L. Lu, S. Jiang, Z. Yang, Y. Wu, T. Ying, Potent binding of 2019 novel coronavirus spike protein by a SARS coronavirusspecific human monoclonal antibody. bioRxiv 9, 382-385 (2020). doi:10.1080/22221751.2020.1729069

32. B. Carragher, N. Kisseberth, D. Kriegman, R. A. Milligan, C. S. Potter, J. Pulokas, A. Reilein, Leginon: An automated system for acquisition of images from vitreous ice specimens. J. Struct. Biol. 132, 33-45 (2000). doi:10.1006/jsbi.2000.4314 Medline

33. D. Tegunov, P. Cramer, Real-time cryo-electron microscopy data preprocessing with Warp. Nat. Methods 16, 1146-1152 (2019). doi:10.1038/s41592-019-0580-y Medline

34. E. Ramírez-Aportela, J. L. Vilas, A. Glukhova, R. Melero, P. Conesa, M. Martínez, D. Maluenda, J. Mota, A. Jiménez, J. Vargas, R. Marabini, P. M. Sexton, J. M. Carazo, C. O. S. Sorzano, Automatic local resolution-based sharpening of cryo-EM maps. Bioinformatics 36, 765-772 (2020). 10.1093/bioinformatics/btz671 Medline

35. A. Šali, T. L. Blundell, Comparative protein modelling by satisfaction of spatial restraints. J. Mol. Biol. 234, 779-815 (1993). doi:10.1006/imbi.1993.1626 Medline

36. E. F. Pettersen, T. D. Goddard, C. C. Huang, G. S. Couch, D. M. Greenblatt, E. C. Meng, T. E. Ferrin, UCSF Chimera-A visualization system for exploratory research and analysis. J. Comput. Chem. 25, 1605-1612 (2004). doi:10.1002/jcc.20084 Medline

37. P. D. Adams, R. W. Grosse-Kunstleve, L.-W. Hung, T. R. loerger, A. J. McCoy, N. W. Moriarty, R. J. Read, J. C. Sacchettini, N. K. Sauter, T. C. Terwilliger, PHENIX: Building new software for automated crystallographic structure determination. Acta Crystallogr. D Biol. Crystallogr. 58, 1948-1954 (2002). doi:10.1107/S0907444902016657 Medline

38. T. I. Croll, ISOLDE: A physically realistic environment for model building into lowresolution electron-density maps. Acta Crystallogr. D Struct. Biol. 74, 519-530 (2018). doi:10.1107/S2059798318002425 Medline

39. P. Emsley, K. Cowtan, Coot: Model-building tools for molecular graphics. Acta $\begin{array}{lllll}\text { Crystallogr. D Biol. Crystallogr. 60, 2126-2132 (2004). } & \end{array}$ doi:10.1107/S0907444904019158 Medline

40. A. Morin, B. Eisenbraun, J. Key, P. C. Sanschagrin, M. A. Timony, M. Ottaviano, P. Sliz, Collaboration gets the most out of software. eLife 2, e01456 (2013). doi:10.7554/el ife.01456 Medline

41. T. Grant, A. Rohou, N. Grigorieff, cisTEM, user-friendly software for single-particle image processing. eLife 7, e35383 (2018). doi:10.7554/el ife.35383 Medline

\section{ACKNOWLEDGMENTS}

We thank Dr. John Ludes-Meyers for assistance with cell transfection and the rest of the members of the McLellan laboratory for critical reading of the manuscript. We would also like to thank Dr. Aguang Dai from the Sauer Structural Biology Laboratory at the University of Texas at Austin for his assistance with microscope alignment. Funding: This work was supported in part by an $\mathrm{NIH/NIAID} \mathrm{grant} \mathrm{awarded} \mathrm{to} \mathrm{J.S.M.} \mathrm{(R01-Al127521)} \mathrm{and} \mathrm{by} \mathrm{intramural} \mathrm{funding}$ from the National Institute of Allergy and Infectious Diseases (B.S.G). The Sauer Structural Biology Laboratory is supported by the University of Texas College of Natural Sciences and by award RR160023 of the Cancer Prevention and Research Institute of Texas (CPRIT). Author contributions:D.W. collected and processed cryo-EM data. D.W., N.W., and J.S.M built and refined the atomic model. N.W. designed and cloned all constructs. D.W., N.W., K.S.C., J.A.G. and O.A. expressed and purified proteins. D.W., J.A.G., and C-L.H. performed binding studies. B.S.G. and J.S.M. supervised experiments. D.W., B.S.G., and J.S.M. wrote the manuscript with help from all authors. Competing interests: N.W., K.S.C., B.S.G, and J.S.M are inventors on US patent application No. 62/412,703 (Prefusion Coronavirus Spike Proteins and Their Use) and D.W., N.W., K.S.C., O.A., B.S.G, and J.S.M are inventors on US patent application No. 62/972,886 (2019-nCoV Vaccine). Data and materials availability: Atomic coordinates and cryo-EM maps of the reported structure have been deposited in the Protein Data Bank under accession code 6VSB and in the Electron Microscopy Data Bank under accession codes EMD-21374 and EMD-21375. Plasmids are available from B.S.G. under a material transfer agreement with the National Institutes of Health or J.S.M. under a material transfer agreement with The University of Texas at Austin.

\section{SUPPLEMENTARY MATERIALS}

science. sciencemag.org/cgi/content/full/science.abb2507/DC1

Materials and Methods

Figs. S1 to S8

Table S1

Movies S1 and S2

References (32-41)

10 February 2020; accepted 17 February 2020

Published online 19 February 2020

10.1126/science.abb2507 


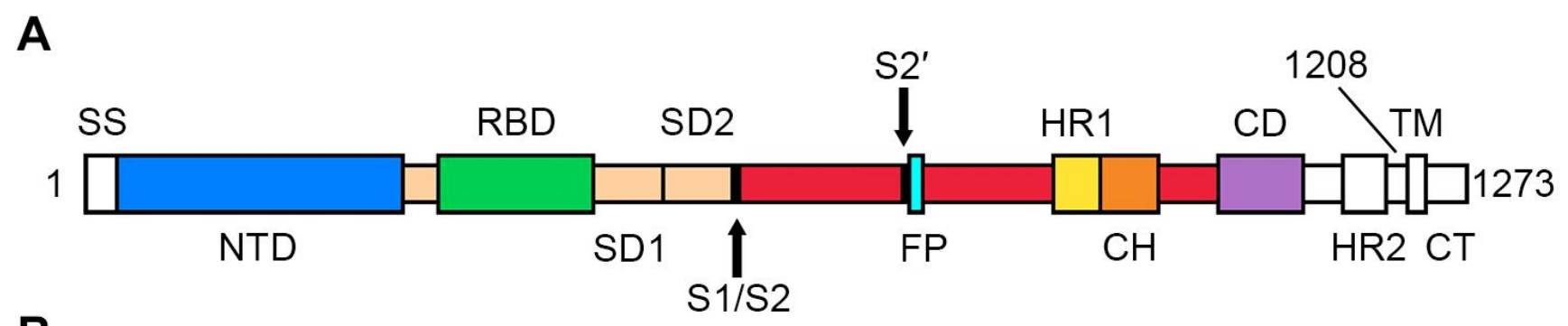

B
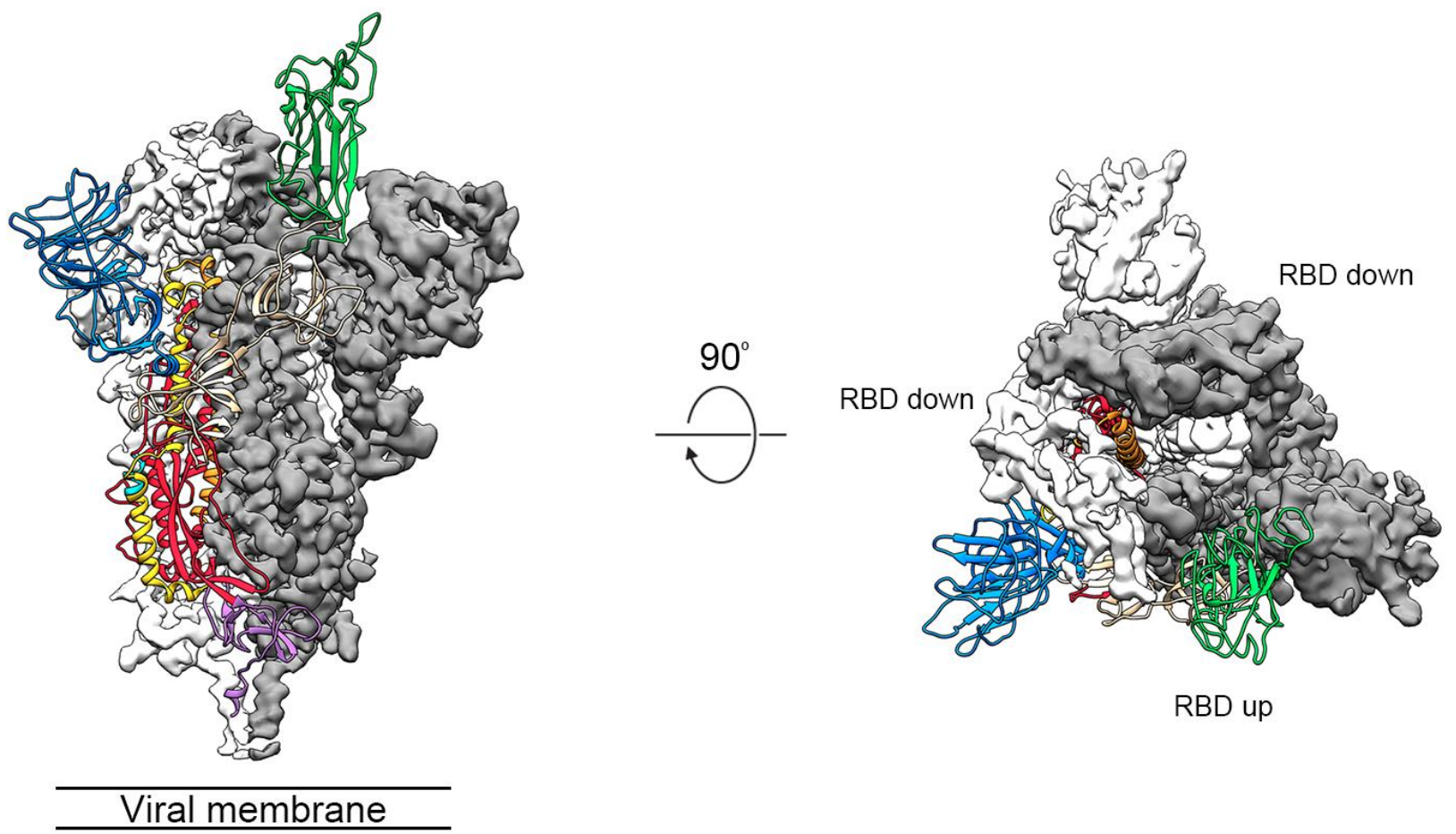

Fig. 1. Structure of 2019-nCoV S in the prefusion conformation. (A) Schematic of 2019-nCoV S primary structure, colored by domain. Domains that were excluded from the ectodomain expression construct or could not be visualized in the final map are colored white. SS= signal sequence, NTD= N-terminal domain, RBD= receptor-binding domain, $\mathrm{SD} 1=$ subdomain $1, \mathrm{SD} 2=$ subdomain $2, \mathrm{~S} 1 / \mathrm{S} 2=\mathrm{S} 1 / \mathrm{S} 2$ protease cleavage site, $\mathrm{S} 2$ ' $=\mathrm{S} 2$ ' protease cleavage site, $\mathrm{FP}=$ fusion peptide, $\mathrm{HR} 1=$ heptad repeat $1, \mathrm{CH}=$ central helix, $\mathrm{CD}=$ connector domain, $\mathrm{HR} 2=$ heptad repeat 2 , $\mathrm{TM}=$ transmembrane domain, $\mathrm{CT}=$ cytoplasmic tail. Arrows denote protease cleavage sites. (B) Side and top views of the prefusion structure of the 2019-nCoV S protein with a single RBD in the up conformation. The two RBD-down protomers are shown as cryo-EM density in either white or gray and the RBD-up protomer is shown in ribbons, colored corresponding to the schematic in $(A)$. 
A
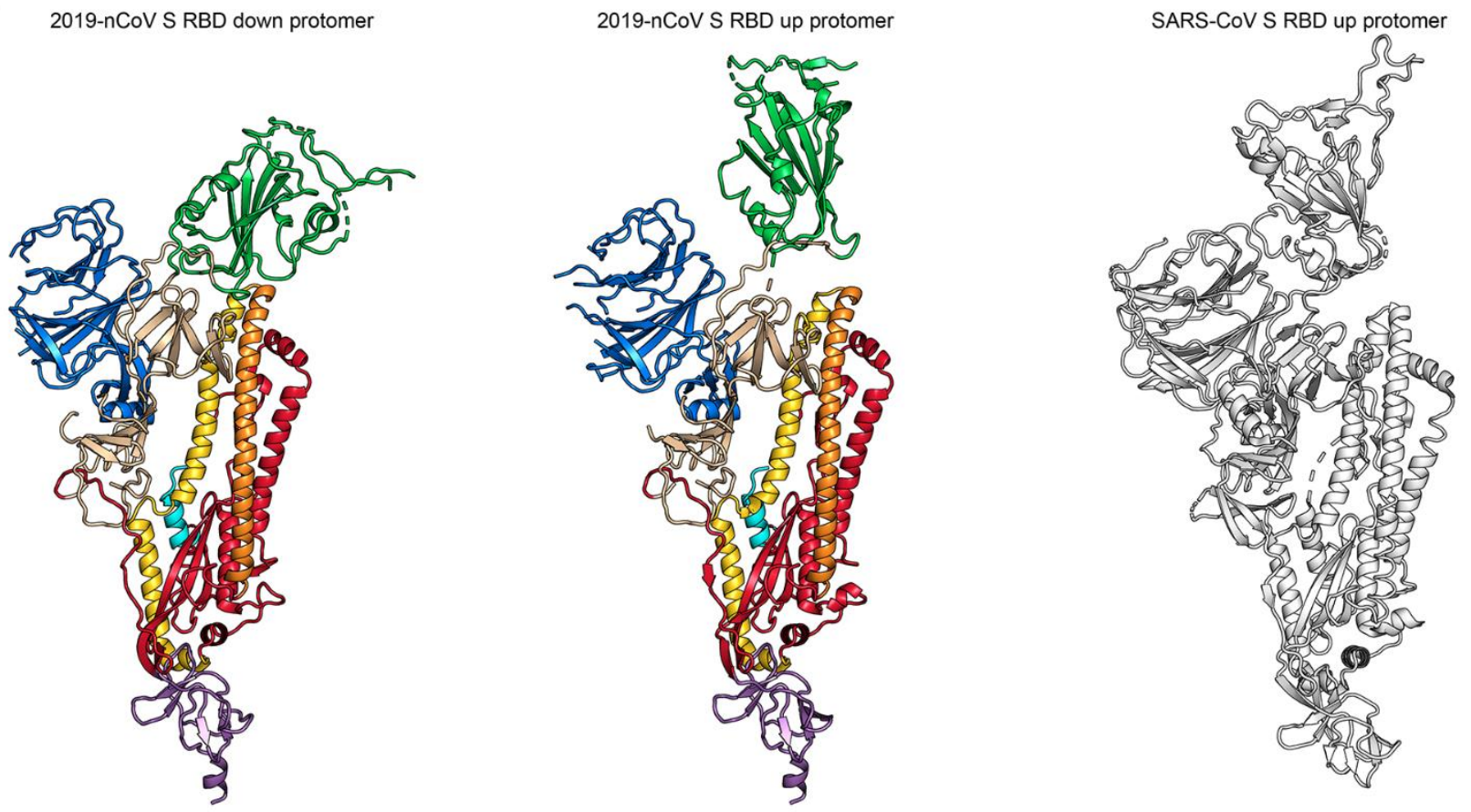

B

C
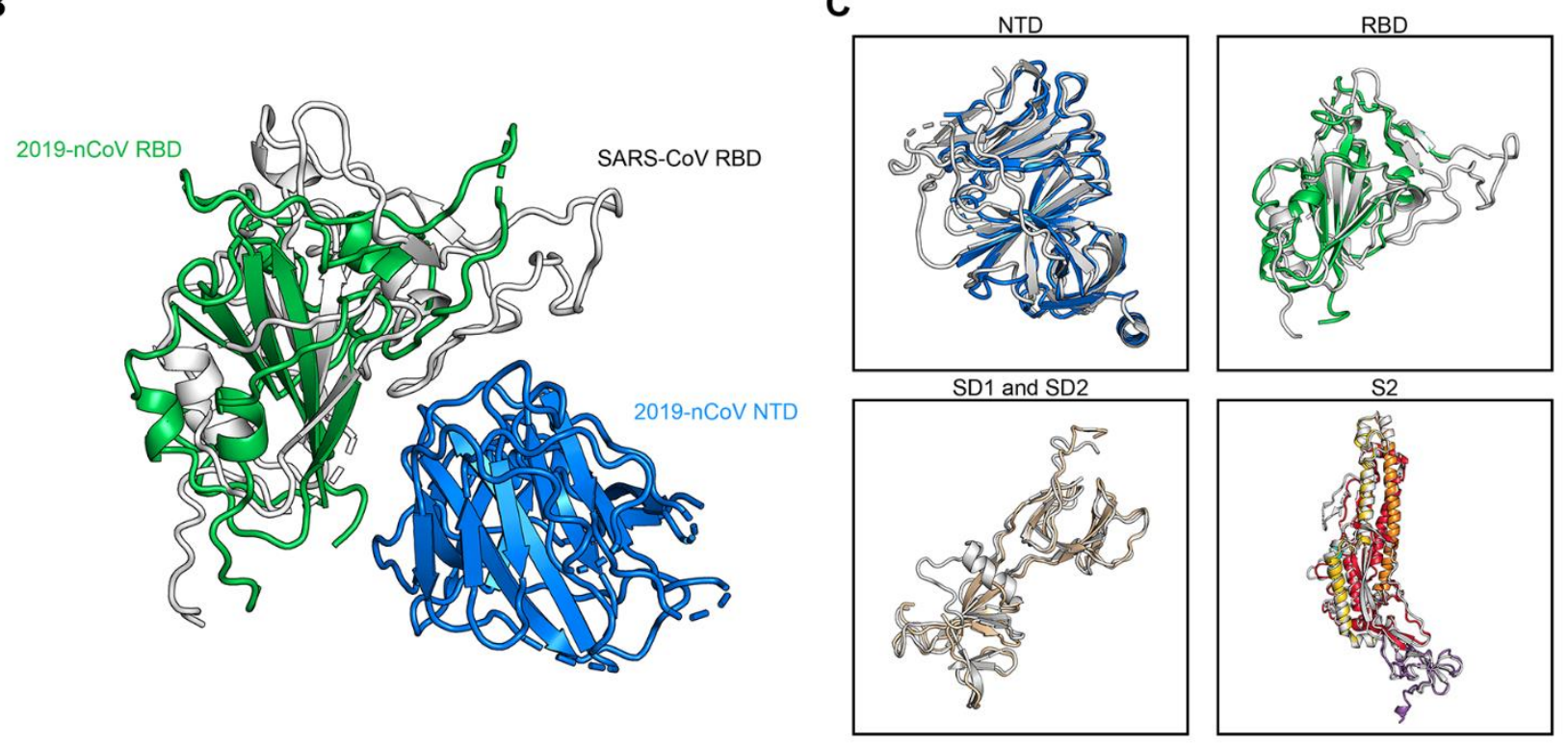

Fig. 2. Structural comparison between 2019-nCoV S and SARS-CoV S. (A) A single protomer of 2019-nCoV S with the RBD in the down conformation (left) is shown in ribbons, colored according to Fig. 1. A protomer of 2019-nCoV S in the RBD-up conformation is shown (center) next to a protomer of SARS-CoV S in the RBD-up conformation (right), displayed as ribbons and colored white (PDB ID: 6CRZ). (B) The RBDs of 2019-nCoV and SARS-CoV have been aligned based on the position of the adjacent NTD from the neighboring protomer. The 2019-nCoV RBD is colored green and the SARS-CoV RBD is colored white. The 2019-nCoV NTD is colored blue. (C) The following structural domains from 2019-nCoV S have been aligned to their counterparts from SARS-CoV S: NTD (top left), RBD (top right), SD1 and SD2 (bottom left), and S2 (bottom right). 
A

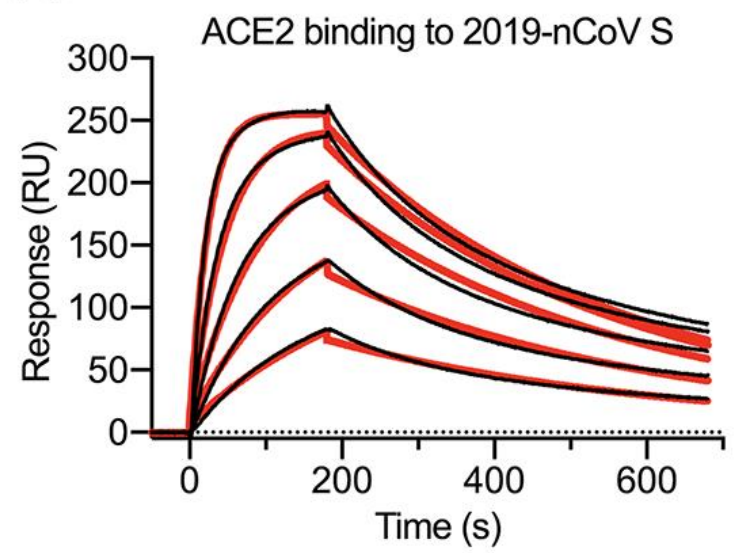

$K_{\mathrm{D}}=14.7 \mathrm{nM}$

$k_{\mathrm{a}}=1.88 \times 10^{5} \mathrm{M}^{-1} \mathrm{~s}^{-1}$

$k_{\mathrm{d}}=2.76 \times 10^{-3} \mathrm{~s}^{-1}$
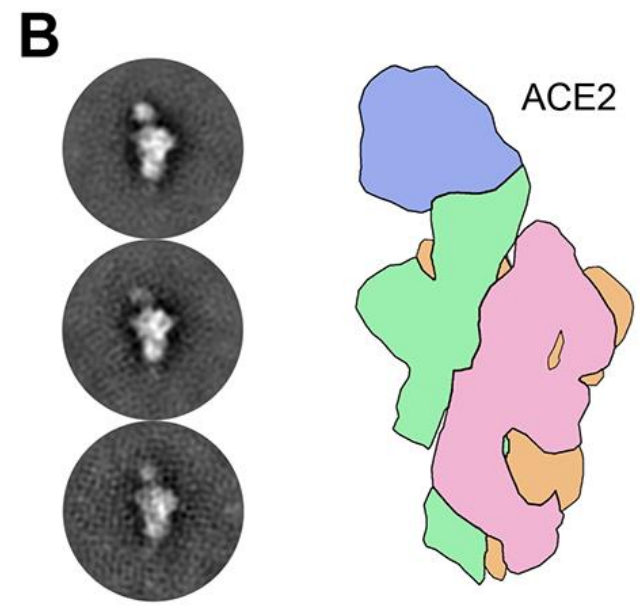

Fig. 3. 2019-nCoV S binds human ACE2 with high affinity. (A) SPR sensorgram showing the binding kinetics for human ACE2 and immobilized 2019-nCoV S. Data are shown as black lines and the best fit of the data to a 1:1 binding model is shown in red. (B) Negative-stain EM 2D class averages of 2019-nCoV S bound by ACE2. Averages have been rotated so that ACE2 is positioned above the 2019-nCoV S protein with respect to the viral membrane. A cartoon depicting the ACE2-bound 2019-nCoV S protein is shown (right) with ACE2 in blue and S protein protomers colored tan, pink and green. 
A

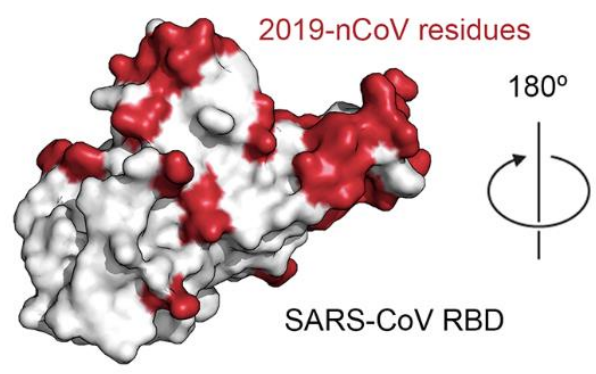

RBD hinge region
ACE2 binding site

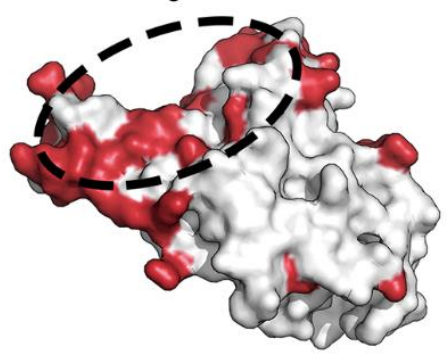

B

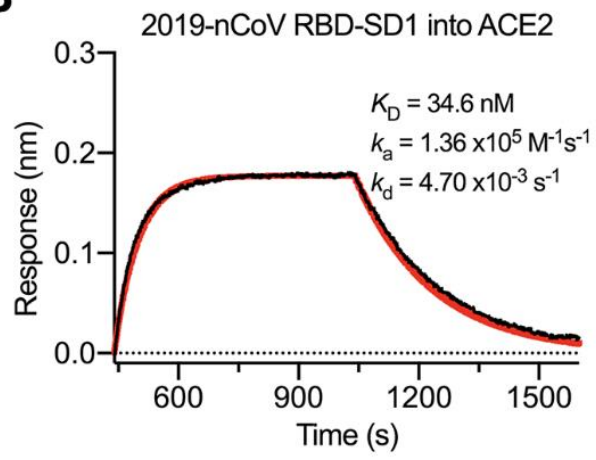

C

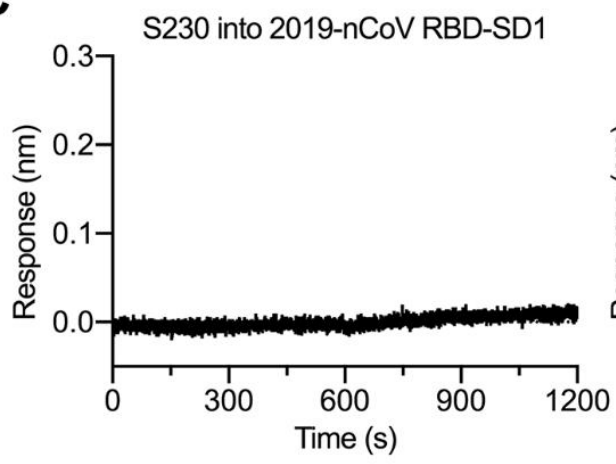

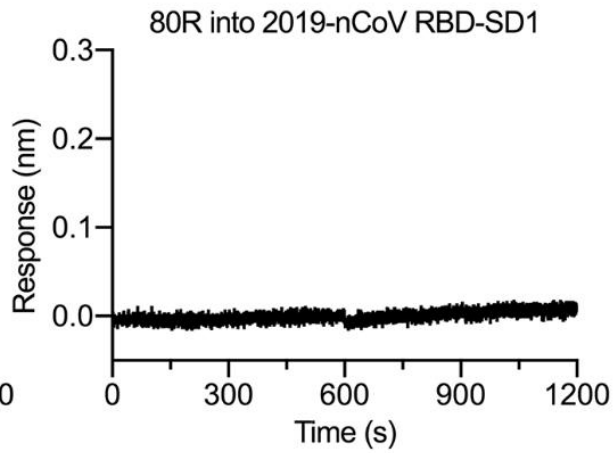

Fig. 4. Antigenicity of the 2019-nCoV RBD. (A) The SARS-CoV RBD is shown as a white molecular surface (PDB ID: 2AJF), with residues that vary in the 2019-nCoV RBD colored red. The ACE2 binding site is outlined with a black dotted line. (B) A biolayer interferometry sensorgram that shows binding to ACE2 by the 2019-nCoV RBD-SD1. Binding data are shown as a black line and the best fit of the data to a 1:1 binding model is shown in red. (C) Biolayer interferometry to measure cross-reactivity of the SARS-CoV RBD-directed antibodies S230, m396 and 80 R. Sensortips with immobilized antibodies were dipped into wells containing 2019-nCoV RBD-SD1 and the resulting data are shown as a black line. 